\title{
Two geometrically frustrated magnets studied by neutron diffraction.
}

\author{
I. Mirebeau, ${ }^{\mathrm{a}, *}$ A. Apetrei, ${ }^{\mathrm{a}}$ I.N. Goncharenko, ${ }^{\mathrm{a}}$ R. Moessner ${ }^{\mathrm{b}}$ \\ ${ }^{a}$ Laboratoire Léon Brillouin, CEA/CNRS UMR12, Centre d'Etudes de Saclay, 91191 Gif sur Yvette, France \\ ${ }^{\mathrm{b}}$ Laboratoire de Physique théorique de l'Ecole Normale Supérieure, CNRS-UMR8549, 75005 Paris, France
}

\begin{abstract}
In the pyrochlore compounds, $\mathrm{Tb}_{2} \mathrm{Ti}_{2} \mathrm{O}_{7}$ and $\mathrm{Tb}_{2} \mathrm{Sn}_{2} \mathrm{O}_{7}$, only the $\mathrm{Tb}^{3+}$ ions are magnetic. They exhibit quite abnormal - and, in view of their chemical similarity, strikingly different - magnetic behaviour, as probed by neutron diffraction at ambient and applied pressure. $\mathrm{Tb}_{2} \mathrm{Ti}_{2} \mathrm{O}_{7}$ is a cooperative paramagnet ('spin liquid'), without long range order at ambient pressure; however, it does become ordered under pressure. By contrast, $\mathrm{Tb}_{2} \mathrm{Sn}_{2} \mathrm{O}_{7}$ enters an "ordered spin ice" state already at ambient pressure. We analyse a simple model which already clearly exhibits some of the qualitative features observed experimentally. Overall, comparing these two compounds emphasizes the power of small perturbations in selecting low-temperature states in geometrically frustrated systems.
\end{abstract}

Key words:

spin liquid, spin ice, neutron diffraction.

\section{Introduction}

Geometrical frustration (GF) [1] is now widely studied in solid state physics, as it seems to play a key role in original phenomena recently observed in new materials. Examples include the large anomalous Hall effect in ferromagnetic pyrochlores or spinels [2], the unconventional superconductivity observed in water substituted $\mathrm{Na}_{x} \mathrm{CoO}_{2}$ with triangular Co sheets [3], or the interaction between electric and magnetic properties of multiferroics materials [4].

What is geometrical frustration? Most simply, it occurs when the specific geometry of the lattice prevents magnetic interactions from being satisfied simultaneously. In insulating systems such as the rare earth pyrochlores, the impossibility of a simple Néel ground state due to GF offers the possibility of finding a large variety of alternative, magnetic and non-magnetic, short- or long-ranged ordered states. In the most ex-

\footnotetext{
* Corresponding Author Email : mirebea@dsmmail.saclay.cea.fr
}

treme case, paramagnetic behaviour persists down to the lowest temperatures, leading to an extended cooperative paramagnetic, or spin liquid, regime, in which only short-range correlations result [5].

Ferromagnetic interactions on the pyrochlore lattice may also be frustrated, namely when the exchange is dominated by a strong anisotropy which forces the spins in a tetrahedron to point along their local, noncollinear easy axes [6]. This leads to the spin ice state, whose degeneracy can be mapped onto that of real ice [6], leading to approximately the same entropy in the ground state [7].

In real compounds, the eventual choice of the stable magnetic state depends on a subtle energy balance between the frustrated first neighbour exchange energy term and perturbation terms of various origins (longer range interactions, anisotropies, quantum fluctuations, ...). It is of course also determined by thermodynamic parameters, such as temperature, pressure or magnetic field. Counterintuitively, thermal fluctuations can even induce order when ordered states permit softer fluctuations than generic disordered ones. This effect is known 
as order by disorder [8] and is commonly encountered in frustrated magnetism. It has been well studied by Monte-Carlo simulations, and also received some experimental confirmation [9]. Pressure can change the nature of, and balance between different terms in the Hamiltonian, as they can depend on interatomic distances in different ways. An applied field adds Zeeman energy, and can, for example, stabilize a subset of the original ground states, at times resulting in magnetization plateaus.

In this paper, we study a well known pyrochlore $\mathrm{Tb}_{2} \mathrm{Ti}_{2} \mathrm{O}_{7}$, which we investigated by neutron diffraction under extreme conditions of temperature (down to $0.1 \mathrm{~K}$ ) and applied pressure (up to $8.7 \mathrm{GPa}$ ). We review one of its most fascinating properties, namely its ability to "crystallize" or order magnetically under pressure and we propose a new theoretical approach which accounts for some important peculiarities of this effect. We also compare $\mathrm{Tb}_{2} \mathrm{Ti}_{2} \mathrm{O}_{7}$ to its sibling compound $\mathrm{Tb}_{2} \mathrm{Sn}_{2} \mathrm{O}_{7}$, very recently studied, which behaves as an "ordered spin ice". Both compounds have a fully chemically ordered structure, the pyrochlore structure of cubic $\mathrm{Fd} \overline{3} \mathrm{~m}$ space group, where the $\mathrm{Tb}^{3+}$ magnetic ions occupy a GF network of corner sharing tetrahedra. Although they differ only by the nature of the nonmagnetic ion $(\mathrm{Ti} / \mathrm{Sn})$, they show very different magnetic ground states. The comparison sheds some light on how to select the ground state through very small perturbations, one of the most prominent characteristics of geometrical frustration.

\section{2. $\mathrm{Tb}_{2} \mathrm{Ti}_{2} \mathrm{O}_{7}$ : a spin liquid orders under applied pressure}

$\mathrm{Tb}_{2} \mathrm{Ti}_{2} \mathrm{O}_{7}$ is a famous example of a spin liquid, investigated by numerous groups, where short range correlated $\mathrm{Tb}$ spins fluctuate down to $70 \mathrm{mK}$ at least [10], that is more than 300 times below the typical energy scale of the magnetic interactions (the Curie-Weiss constant $\theta_{\mathrm{CW}}$ of $-19 \mathrm{~K}$, where the minus sign corresponds to AF interactions). The persistence of these fluctuations was checked by muon relaxation [10], at the time scale of the muon probe of about $10^{-6} \mathrm{~s}$. At shorter time scales, inelastic neutron scattering showed a quasi elastic signal, whose energy linewidth strongly decreases below about $1 \mathrm{~K}$, indicating a stronger slowing down in this temperature range [11]. Coexisting with the spin liquid phase, spin glass like irreversibilities and anomalies of the specific heat were recently observed in the range $0.1 \mathrm{~K}-0.8 \mathrm{~K}[12]$. Using high pressure powder neutron diffraction [13], we observed two interesting phenomena induced by pressure [14] i) the onset of antiferromagnetic long range order below a Néel temperature $\mathrm{T}_{\mathrm{N}}$ of about $2 \mathrm{~K}$ : ii) the enhancement of the magnetic correlations in the spin liquid phase above $\mathrm{T}_{\mathrm{N}}$. Just below $T_{\mathrm{N}}$, the ordered phase coexists with the spin liquid in a mixed solid-liquid phase, whose relative contributions vary with pressure and temperature. The magnetic Bragg peaks of the simple cubic lattice can be indexed from the crystal structure of $\mathrm{Fd} \overline{3} \mathrm{~m}$ symmetry, taking a propagation vector $\mathbf{k}=(1,0,0)$. It means that in the cubic unit cell with four Tb tetrahedra, two tetrahedra are identical and two have reversed moment directions. A longer wavelength modulation of this structure, involving a much larger unit cell, was also observed in the powder data.

What is the pressure induced ground state? More fundamentally what is the role of pressure? To answer these questions, we performed single crystal neutron diffraction down to very low temperatures $(0.14 \mathrm{~K})$, combining hydrostatic pressure with anisotropic stress [15]. We showed that both components play a role in inducing the long range order, and that the ordered moment and Néel temperature can be tuned by the direction of the stress. A stress along a [110] axis, namely along the direction of the first neighbour distances between $\mathrm{Tb}^{3+}$ ions, is the most efficient in inducing magnetic order (Fig. 1).

FullProf refinements of the single crystal data allowed a determination of the magnetic structure with better precision, especially the local spin structure within a $\mathrm{Tb}$ tetrahedron. The structure corresponding to the best refinement $\left(\mathrm{R}_{F}=14 \%\right.$ is given in table 1 . The bond 1-4 along the axis of the stress, which should be reinforced, has AF collinear spins. This corresponds to a natural expectation for AF first neighbor exchange. The orientation of the spin 2 (orthogonal to the 3 others) is more surprising since with 3 collinear spins, the exchange field on the fourth one should be also collinear. Since no collinear structure gave a good fit to the data, it suggests that the real spin structure may be even more complex than the proposed one. In any case, both powder and single crystal data yield an important conclusion: we found that inside a tetrahedron, the magnetization is not compensated, namely the vectorial sum of the four spins is non zero, (although it is of course compensated within the cubic

\begin{tabular}{ccccc}
\hline site & $x$ & $y$ & $z$ & $M$ \\
\hline 1 & 0.5 & 0.5 & $0.5\left[\begin{array}{lll}1 & 0 & -1\end{array}\right]$ \\
2 & 0.25 & 0.25 & 0.5 & {$\left[\begin{array}{lll}1 & 0 & 1\end{array}\right]$} \\
3 & 0.25 & 0.5 & $0.25\left[\begin{array}{lll}-1 & 0 & 1\end{array}\right]$ \\
4 & 0.5 & 0.25 & $0.25\left[\begin{array}{lll}-1 & 0 & 1\end{array}\right]$ \\
\hline
\end{tabular}

Table 1

Orientation of the magnetic moments in one tetrahedron in the pressure induced state of Fig 1., deduced from the re inement of the magnetic structure. The stress component is along [ $\left[\begin{array}{ll}0 & 1\end{array}\right.$ $1]$. The atomic coordinates $x, y, z$, are expressed in the cubic unit cell containing 4 tetrahedra. Two tetrahedra are identical and two have reversed spin orientations. 


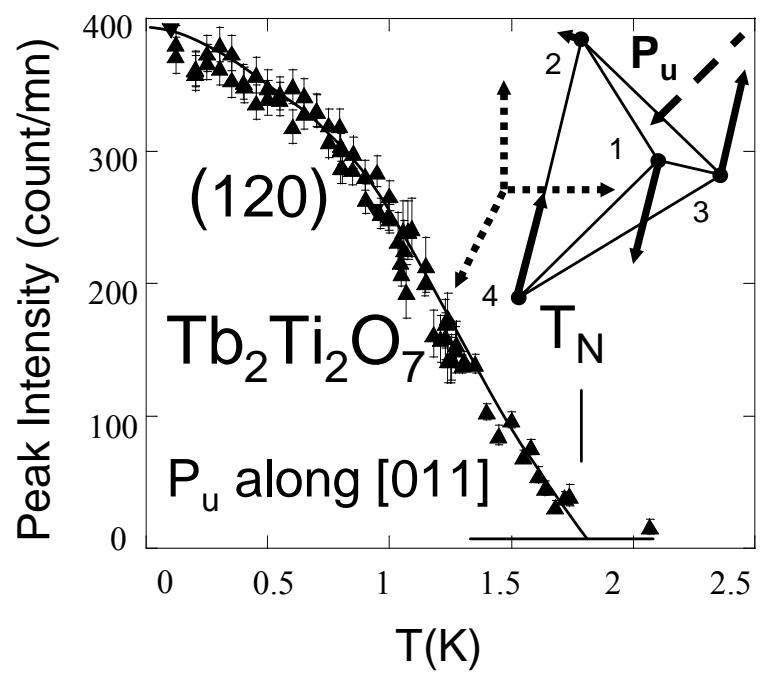

Fig. 1. $\mathrm{Tb}_{2} \mathrm{Ti}_{2} \mathrm{O}_{7}$ : an antiferromagnetic ordered state with $\mathbf{k}=(1,0,0)$ propagation vector is induced under pressure. Here an isotropic pressure $\mathrm{P}_{i}=2.0 \mathrm{GPa}$ is combined with uniaxial pressure $\mathrm{P}_{u}=0.3 \mathrm{GPa}$ along [011] axis. The variation of the peak intensity of the magnetic Bragg peak (120) shows the Néel temperature. The local spin structure in a tetrahedron has non compensated magnetization.

cell, since magnetisations of the four tetrahedra cancel two by two). This means that in the pressure induced ground state, the local order does not correspond to any configuration which mimimizes the energy in the spin liquid phase. In other words, pressure does not select any energy state among those belonging to the ground state degeneracy of the spin liquid (the ground state expected if one considers Heisenberg spins coupled via first neighbour AF exchange interactions only). The anisotropic component (stress) relieves the frustration in a more drastic way, by creating uncompensated bonds, associated with a very small distortion of the pyrochlore lattice. In addition the isotropic component shortens all distances in the same way, increasing the frustrated exchange interaction. This effect could also contribute to the increase of $\mathrm{T}_{\mathrm{N}}$.

The prominent role of stress in inducing magnetic order raises a subsequent question. Could it be stabilized spontaneously by internal stresses? To answer this question, we have now checked the magnetic order at ambient pressure by neutron diffraction in two $\mathrm{Tb}_{2} \mathrm{Ti}_{2} \mathrm{O}_{7}$ samples with different heat treatments, down to about $0.1 \mathrm{~K}$. In an "as cast" powder sample, we observe at $0.07 \mathrm{~K}$ broad magnetic peaks close to the positions expected for the pressure induced magnetic order (Fig. 2). The Lorentzian lineshape corresponds to a finite correlation length of about $25 \AA$ (2-3 cubic cells). The peaks disappear around $0.3 \mathrm{~K}$. We also studied a single crystal, which was annealed at 1150 ${ }^{\circ} \mathrm{C}$ for 25 hours to relieve internal stresses. In the second case, the mesoscopic magnetic order is absent and

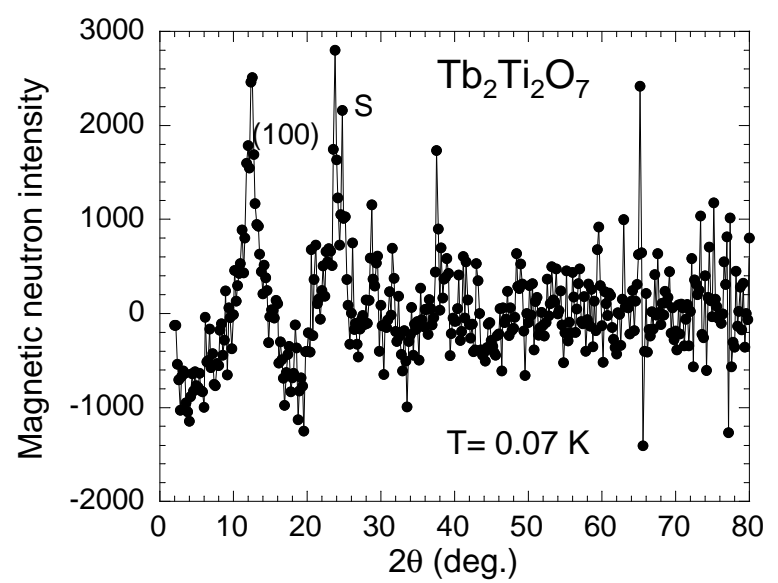

Fig. 2. $\mathrm{Tb}_{2} \mathrm{Ti}_{2} \mathrm{O}_{7}$ : a mesoscopic order is induced by spontaneous strains at very low temperature. Magnetic neutron diffraction spectra at $0.07 \mathrm{~K}$, showing broad peaks close to the positions of the (100) magnetic peak and secondary magnetic phase (S) of the pressure-induced state [14]. The spectrum of the spin liquid regime at $1.2 \mathrm{~K}$ has been subtracted. The incident neutron wavelength is $2.52 \AA$. The broad magnetic peaks disappear at about $0.3 \mathrm{~K}$.

only the liquid-like correlations are observed, down to the minimum temperature of $0.15 \mathrm{~K}$. Since both samples are chemically ordered and stoichiometric within the accuracy of neutron diffraction, it means that the mesoscopic order is induced by internal stresses. The onset of this mesoscopic order may strongly influence the spin glass irreversibilities and anomalies of the specific heat observed in the same temperature range[12], which seem to depend on the heat treatment.

\section{3. $\mathrm{Tb}_{2} \mathrm{Sn}_{2} \mathrm{O}_{7}$ : an ordered spin ice state}

In contrast to $\mathrm{Tb}_{2} \mathrm{Ti}_{2} \mathrm{O}_{7}, \mathrm{~Tb}_{2} \mathrm{Sn}_{2} \mathrm{O}_{7}$ undergoes a transition to an ordered state already at ambient pressure. The magnetic structure, very recently determined by powder neutron diffraction experiments [16] was called an "ordered spin ice". The local order within one tetrahedron is close to the "two in-two out" configuration of spin ice, taking into account a small deviation of $13^{\circ}$. of the magnetic moments with respect to the local $<111>$ easy anisotropy axes. In the canonical spin ice state, individual tetrahedra keep the mutual orientational disorder allowed by the "ice rules", leading to short range order and ground state entropy [17]. Here the four tetrahedra of the unit cell are identical, leading to an ordered structure with $\mathbf{k}=0$ propagation vector (Fig. 3). The resulting magnetic structure is non-collinear, but exhibits a ferromagnetic component, which represents about $37 \%$ of the $\mathrm{Tb}^{3+}$ ordered moment. This explains the ferromagnetic character of the transition, previously observed by magnetization 


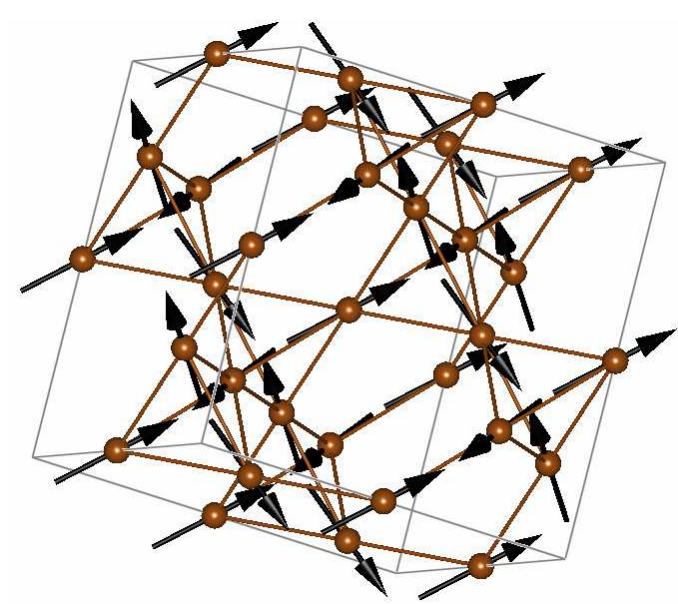

Fig. 3. $\mathrm{Tb}_{2} \mathrm{Sn}_{2} \mathrm{O}_{7}$ : an ordered spin ice structure: the local spin structure in a tetrahedron is close to the " 2 in- 2 out" structure of a spin ice, but individual tetrahedra are identical, leading to an ordered structure with $\mathbf{k}=0$ propagation vector and ferromagnetic character.

\section{[18].}

Together with the non-collinear magnetic structure, the original effects of the frustration persist in the ordered phase of $\mathrm{Tb}_{2} \mathrm{Sn}_{2} \mathrm{O}_{7}$. The magnetic order is stabilized in two steps $(1.3 \mathrm{~K}$ and $0.87 \mathrm{~K})$ corresponding to anomalies of the specific heat, and not in a classical second order transition. The correlation length increases throughout the transition region, and remains limited to $180 \AA$ even at very low temperature. The ordered state coexists with slow collective fluctuations, in the time scale of $10^{-4}-10^{-5} \mathrm{~s}$. They were probed by comparing the $\mathrm{Tb}^{3+}$ moment value of 5.9(1) $\mu_{B}$ deduced from neutron diffraction to the much lower value of 3.3(3) $\mu_{B}$ deduced from the specific heat.

The magnetic order in $\mathrm{Tb}_{2} \mathrm{Sn}_{2} \mathrm{O}_{7}$ may be compared to that found by Champion et al. [19], who considered the competition between first neighbor exchange and uniaxial anisotropy in a pyrochlore ferromagnet. The model involves two parameters, the strength of the ferromagnetic interaction $\mathrm{J}$ and that of the uniaxial anisotropy $\mathrm{D}_{\mathrm{a}}$ along $<111>$ axes. Ferromagnetic and spin ice states correspond to the cases $\mathrm{D}_{\mathrm{a}} / \mathrm{J}=0$ and $D_{a} / J=\infty$, respectively. For finite $D_{a} / J$ values, the magnetic order shows many similarities with the observed one. Namely: i) the ground state is ordered in a $\mathbf{k}=0$ four sublattice structure. ii) the local order within one tetrahedron may also be deduced from the spin ice structure. iii) the magnetic transition is of first order, changing to second order with decreasing $\mathrm{D}_{\mathrm{a}} / \mathrm{J}$.

However, the deviations from the local spin ice structure are different in the model and in the real system. In the model, spins are uniformly canted towards the ferromagnetic direction. The ground state magnetization relative to the local moment increases from
$0.578=1 / \sqrt{3}$ (the average magnetization of a tetrahedron in the spin ice case) to 1 (the ferromagnetic case) with decreasing $\mathrm{D}_{\mathrm{a}} / \mathrm{J}$. By contrast, in $\mathrm{Tb}_{2} \mathrm{Sn}_{2} \mathrm{O}_{7}$, the deviations of the magnetic moments from the local $<111>$ axes actually reduce the magnetization (to about 0.37 in relative units). So the deviations of the magnetic moments from the local spin ice structure act in an opposite way to that predicted by the finite anisotropy ferromagnetic model.

Finally, in $\mathrm{Tb}_{2} \mathrm{Sn}_{2} \mathrm{O}_{7}$, the neutron and magnetic data together with the comparison with theory, suggest that here the effective first neighbor interaction becomes ferromagnetic, although the physics of the system cannot be simply reduced to the energy scheme assumed in ref. [19].

\section{A simple model for the influence of stress}

An analysis of the effect of pressure applied to an individual tetrahedron already manifests qualitatively two basic experimental results observed in $\mathrm{Tb}_{2} \mathrm{Ti}_{2} \mathrm{O}_{7}$ : the much stronger influence of an anisotropic stress along a [110] axis (as opposed to hydrostatic pressure or to a stress along the [100] direction), and the presence of an uncompensated magnetisation.

In the isotropic problem, all six bonds of the tetrahedron are equivalent. Application of stress in the [110] direction lowers this symmetry, as shown in Fig. 4. In symmetry terms, the bonds form a six-dimensional representation of the tetrahedral group $T_{d}$, which decomposes into three irreducible representations. A singlet, A amounts to a uniform change of all bonds together. Furthermore, there is a doublet, E, the components of which correspond (a) to strengthening two opposite bonds, and weakening the four others (or vice versa) or (b) to weakening an opposite pair of those four bonds, and strengthening the other pair. Finally, each component of the triplet, $\mathrm{T}$, implies a strengthening/weakening (by an equal amount) of an opposite pair of bonds [20].

The crucial point is that the uniaxial [110] stress can couple to all three representations. In its presence, there are three (instead of only one) symmetryinequivalent bond strengths (see Fig. 4). In other words, the Hamiltonian including the uniaxial [110] stress has a lower symmetry than the isotropic one. The case of $\left[\begin{array}{lll}1 & 0 & 0\end{array}\right]$ pressure is intermediate: here only two representations are present, the triplet being absent, as illustrated in the left panel of Fig. 4.

Whereas the initial degeneracy of the isotropic system is a signature of the different possible compromises between which bonds to frustrate and which to satisfy, some of these choices have become forbidden as it is not possible to trade off inequivalent bonds against one 


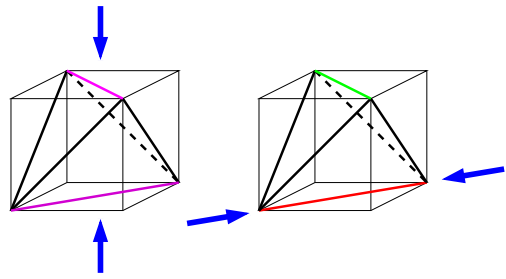

Fig. 4. Tetrahedron under uniaxial stress, denoted by the arrows. Left (right) panel: stress applied in the [100] ([110]) direction. This splits the six bonds into the following symmetry-inequivalent groups: the bond along the [110] direction (bottom), the one perpendicular to it (top) - which remain equivalent for [100] but not for [110] stress - and the four remaining ones.

another.

For simplicity, let us consider a classical, isotropic Heisenberg antiferromagnet at $\mathrm{T}=0$ under stress. We have considered the spin configurations which minimize the energy for different orientations of the stress. We find that these configurations have a compensated magnetization for a stress along [100]. By contrast, a non-compensated magnetic moment can arise for a stress along a [110] axis. A summary of the calculation is given below.

The results for an ice-type model (i.e. a ferromagnet in the presence of anisotropy $\mathrm{D}_{\mathrm{a}}$ ) can be obtained along similar lines. It needs to be borne in mind, however, that (a) the strict ice model $\left(\mathrm{D}_{\mathrm{a}} / \mathrm{J}=\infty\right)$ does not permit small deviations of the spins from their preferred axes, and that (b) a ferromagnet will generically exhibit a non-compensated moment even in the absence of stress. By contrast, an anisotropic (but strainfree) antiferromagnet has a momentless ground state, which, however, is the simple $\mathrm{FeF}_{3}$-type 'all-in' or 'allout' ground state.

This classical isotropic Hamiltonian has a continuous two-parameter family of degenerate ground states in the isotropic case [1]. This degeneracy is reduced by the strain. For example, in the presence of an E-distortion weakening the average strength of the top/bottom pair of bonds with respect to the other two pairs, a collinear state will be selected. In this state, each spin is parallel to its partner at the other end of the coloured bond, and antiparallel to the other pair of spins. The total spin of the tetrahedron thus remains compensated at zero. For an $E$ distortion of the opposite sign, the top (and bottom) pair of spins will be antialigned; for the full pyrochlore lattice, this generates decoupled chain states. In contrast to this situation, the presence of a $T$ distortion does not change the energy of the isotropic ground states relative to one another to first order. This happens because it couples to a difference in the expectation value of opposing bonds, a difference which vanishes in the unperturbed ground states.

However, in higher order, a difference in relative bond strength can induce a difference between the expectation values of the scalar product $S_{i} \cdot S_{j}$ across the bottom and top bonds. Such a difference is equivalent to an uncompensated total moment of the tetrahedron (as a tetrahedron with zero moment necessarily has equal expectation values of $S_{i} \cdot S_{j}$ on opposite bonds). The ground states in the presence of stress are thus close to - but not a subset - of those of the isotropic system.

\section{Discussion}

In this section we briefly comment about the relevance of the above model to $\mathrm{Tb}_{2} \mathrm{Ti}_{2} \mathrm{O}_{7}$, and then focus on the origin of the differences between the two compounds.

At ambient pressure, the fact that $\mathrm{Tb}_{2} \mathrm{Ti}_{2} \mathrm{O}_{7}$ does not order but remains 'liquid' down to $70 \mathrm{mK}$, is still a challenge to theory. Sophisticated calculations taking into account the crystal field energy [21] together with dipolar interactions, predict an Ising like behavior for the $\mathrm{Tb}$ ion moment in the the ground state (with the moment reduced with respect to the free ion value) and an effective AF first neighbor interaction[22]. These calculations predict at ambient pressure a transition to an $\mathrm{AF}$ order similar to that found in $\mathrm{FeF}_{3}$ (a $\mathbf{k}=0$ structure with an "all in-all out" local configuration) below $1-2 \mathrm{~K}$, which is however not what is observed experimentally.

The simple model discussed above, already proposed on a more empirical basis in Ref. [16], reproduces the main characteristics of the pressure-induced state in $\mathrm{Tb}_{2} \mathrm{Ti}_{2} \mathrm{O}_{7}$, namely the stronger effect of degeneracy lifting of the [110] over the [100] stress, and the appearance of an uncompensated magnetisation in the former case. This is presumably the case because it incorporates the most fundamental property of the stress, namely the explicit symmetry breaking it induces. This effect should occur in qualitatively the same way in a much larger class of models.

These results are therefore rather robust but, by the same token, they are also only qualitative: the model in its current form yields little information on the detailed Hamiltonian of the system, nor the origin of the effective nearest-neighbour interaction $\mathrm{J}$ and of its sensitivity to pressure. In particular, we have not been able to reproduce the detailed finite-temperature spin structure.

We now turn to $\mathrm{Tb}_{2} \mathrm{Sn}_{2} \mathrm{O}_{7}$, which behaves as an ordered spin ice. Neutron data as compared with theory strongly suggest that the effective first neighbour interaction has now become ferromagnetic. What is the reason for this change? We can propose the following explanation. In the "true" spin ices $\left(\mathrm{Ho}_{2} \mathrm{Ti}_{2} \mathrm{O}_{7}\right.$ or 
$\mathrm{Dy}_{2} \mathrm{Ti}_{2} \mathrm{O}_{7}$ with stronger uniaxial anisotropy), it was shown that the effective ferromagnetic interaction results from the influence of the dipolar coupling which overcomes the weak AF superexchange [17]. Taking the same conventional notations, the effective first neighbour interaction $\mathrm{J}_{e f f}$ is expressed as $\mathrm{J}_{e f f}=\mathrm{J}_{n n}+\mathrm{D}_{n n}$, where $\mathrm{J}_{n n}=\mathrm{J} / 3$ and $\mathrm{D}_{n n}=5 \mathrm{D} / 3$ are the superexchange and dipolar energy scales, respectively. In $\mathrm{Tb}_{2} \mathrm{Ti}_{2} \mathrm{O}_{7}$ $\left(\mathrm{J}_{n n}=-0.88 \mathrm{~K}, \mathrm{D}_{n n}=0.8 \mathrm{~K}\right.$ from ref. [22]), this effective interaction remains AF. In $\mathrm{Tb}_{2} \mathrm{Sn}_{2} \mathrm{O}_{7}$, Sn substitution enlarges the unit cell (from $\mathrm{a}=10.149$ to $10.426 \AA$ in $\mathrm{Ti}$ and Sn compounds respectively). This expansion $\Delta a / a \sim 2.7 \%$, equivalent to a negative chemical pressure of about 12-15 GPa, should strongly decrease the AF superexchange interaction J. Assuming roughly a decrease of $\mathrm{J}_{n n}$ in the ratio of the Curie-Weiss constants $(-19 \mathrm{~K}$ and $-12 \mathrm{~K}$ in $\mathrm{Ti}$ and $\mathrm{Sn}$ compounds respectively) without big changes in the dipolar constant, we get $\mathrm{J}_{\text {eff }}=0.18 \mathrm{~K}>0$ for $\mathrm{Tb}_{2} \mathrm{Sn}_{2} \mathrm{O}_{7}$. Therefore the expansion in the unit cell induced by $\mathrm{Sn}$ substitution might be enough to switch the compound to the spin ice region of the phase diagram [23].

To go further, microscopic models should take into account the exact nature of the anisotropy, which is not simply uniaxial in the Tb compounds[21]. This involves a reinvestigation of the crystal field levels, currently in progress. It could exhibit more subtle differences between the two compounds than the simple effect of a chemical pressure discussed above.

In conclusion, the two compounds studied here clearly show the rich variety of behaviour exhibited by geometrically frustrated magnets. Comparing them allows one to understand better the key role played by small perturbations in selecting one peculiar state among the many potential magnetic states.

We thank A. Gukasov and O. Isnard for their help in the neutron measurements at LLB and ILL respectively. We also thank G. Dhalenne, A. Revcolevschi, A. Forget and D. Colson, who provided the single crystal and powdered samples. R. M. thanks S. Sondhi and O. Tchernyshyov for collaboration on related work. He was supported in part by the Ministère de la Recherche with an ACI grant.

\section{References}

[1] For an introduction to frustrated magnets, see $R$. Moessner, Can. J. Phys. 79, 1283, (2001); reviews of exact diagonalizations and experiments, respectively, are C. Lhuillier, P. Sindzingre and J.-B. Fouet, Can. J. Phys. 79, 1525, (2001) and P. Schiffer and A. P. Ramirez, Comments Cond. Mat. Phys. 18, 21, (1996).

[2] Y. Taguchi, Y. Oohara, H. Yoshisawa, N. Nagaosa, Y. Tokura Science 291, 2573 (2001).
[3] K. Takada et al. Nature 422, 53, (2003).

[4] G. R. Blake et al., Phys. Rev. B. 71, 214402, (2005).

[5] J. Villain Z. Phys. B 33, 31; (1979).

[6] M. J. Harris et al., Phys. Rev. Lett. 79, 2554 (1997).

[7] A. P. Ramirez, A Hayashi, R. J. Cava, R. Siddhartan, B.S. Shastry Nature, 399, 333, (1999).

[8] J. Villain, R. Bidaux, J. P. Carton, R. Coute J. Phys. (Paris) 41, 1263, (1980); E. F. Shender Sov. Phys. JETP 56, 178, (1982).

[9] A. G. Gukasov et al., Europhys. Lett. 79, 2554 (1997); J. D. M. Champion et al., Phys. Rev. B 68, 020401 R (2003)

[10] J. S. Gardner et al. Phys. Rev. Lett. 82, 1012, (1999).

[11] Y. Yasui et al., J. Phys. Soc. Jpn. 71, 599, (2002).

[12] N. Hamaguchi, T. Matsushita, N. Wada, Y. Yasui and S. Masatoshi, Phys. Rev. B 69, 132413, (2004).

[13] I. N. Goncharenko, High Pressure Res. 24,193,(2004).

[14] I. Mirebeau, I.N. Goncharenko, P.Cadavez-Peres, S. T. Bramwell, M.J.P. Gingras and J. S. Gardner Nature 420, $54,(2002)$.

[15] I. Mirebeau, I. N. Goncharenko, G. Dhalenne, A. Revcolevschi, Phys. Rev. Lett. 93, 187204, (2004); I. Mirebeau and I. Goncharenko J. Phys. Cond. Mat. 17, S771, (2005).

[16] I. Mirebeau et al. Phys. Rev. Lett. 94, 246402, (2005).

[17] S. T. Bramwell and M. J. P. Gingras Science 294, 14, (2001)

[18] K. Matsuhira et al. J. Phys. Soc. Jpn. 71,1576,(2002).

[19] J. D. M. Champion, S. T. Bramwell, P. C. W. Holdsworth and M. J. Harris, Europhys. Lett. 57, 93 (2002).

[20] Y. Yamashita and K. Ueda, Phys. Rev. Lett. 85, 4960 (2000); O. Tchernyshyov, R. Moessner, S. L. Sondhi, Phys. Rev. Lett. 88, 067203 (2002).

[21] M. J. P. Gingras et al. Phys. Rev. B 62, 6496, (2000).

[22] Y. Kao, M. Enjalran, A. Del Maestro, H. Molavian and M. J. P. Gingras Phys. Rev. B 68, 172407, (2002); M. Enjalran and M. J. P. Gingras Phys. Rev. B 70, 174426, (2004).

[23] B. C. Den Hertog and M. J. P. Gingras Phys. Rev. Lett. 84, 3430, (2000). 